\title{
A Numerical Analysis of Double-Aperture Interferences
}

\author{
Youssef Khmou ${ }^{1}$, Said Safi ${ }^{2}$ and Miloud Frikel ${ }^{3}$ \\ ${ }^{1,2}$ Department of Mathematics and Informatics, \\ Sultan Moulay Slimane University, Morocco \\ ${ }^{3}$ GREYC Lab UMR 6072 CNRS, Equipe Automatique, \\ Caen, France \\ ${ }^{1}$ khmou.y@gmail.com, ${ }^{2}$ safi.said@gmail.com, ${ }^{3}$ mfrikel@greyc.ensicaen.fr
}

\begin{abstract}
This paper treats the problem of diffraction of monochromatic field by two apertures under the conditions of normal incidence and linear polarization. We study the effect of diffraction and interferences by two thin apertures in the Fraunhofer region using Gauss approximation, where we introduce a birefringent wedge whose length is equal to the width of single aperture, we demonstrate the effect of the wedge's thickness on the intereference pattern.
\end{abstract}

Keywords: Double aperture, Diffraction, Interferences, Intensity distribution, Birefringence.

\section{Introduction}

The electromagnetic field originating from an aperture whose dimension is comparable to the wavelength of the field is diffracted [1], this effect is studied in both near field and far field regions. For the second case, the expression of the electric field is in general a function of the Fourier transform of the spatial response of the aperture [2], this remarkable effect is based on the same concept of frequency analysis, when a periodic waveform is analyzed in limited period of time or window, the frequency spectrum is function of the expression of the observation window (an illustrative example is given in the appendix). In the case of two apertures [3], the expression of the electric field is combination of two effects, diffraction and interferences, where the intensity distribution when observed on screen placed in the far field region is characterized by an amplitude modulation, the intensity has slow and fast frequencies where the first is related to the width of the aperture and the second frequency is function of distance between the apertures.

Since the discovery of wave-particle nature of visible spectrum of electromagnetic fields, the diffraction was also observed for particles like electrons [4], where the diffraction and interference patterns follow the same model of the electromagnetic waves. The distribution of the incident electrons on screen is proportional to the square of the wave function which is also the probability density function. Given the effect of interference that governs the shape of the intensity distribution, altering the pattern is possible by controlling the phase difference of the propagating waves either from the source, between the source and the diffraction plan, or right after the diffraction plan where the phase shifting consists of changing the polarization state [5], by using retarders [6], by the effect of dichroism, or by birefringent materials [7].

In this paper, we treat the problem of the diffraction and interferences by two thin apertures in far field region, we study the problem with new configuration using birefringent wedge $[7,8,9,10]$ that is material characterized by slow and fast axis of

Received (February 5, 2018), Review Result (May 1, 2018), Accepted (May 11, 2018) 
propagation. We study the intensity function based single mode laser field that vertically polarized, with normal incidence using Gauss approximation. Based on numerical simulations, we demonstrate that the introduction of the birefringent wedge whose length equals the width of single aperture, changes the shape of the intensity distribution.

\section{Standard Double Aperture Interferences}

In this section, we present a description of standard double aperture problem using single mode laser with normal incidence, let us consider that the plan of diffraction is $(o, x, y)$ where the incident electric field is vertically polarized such as the variation of the amplitude is parallel to $z$ axis and the wave vector $\vec{k}$ is parallel to $y$ axis. The diffraction plan consists of two thin vertical apertures with width $a$ and height $b$ such that $b>>a$, the distance between the centers of the apertures is $d$. We begin the analysis of the diffracted field with the first aperture, a screen is placed perpendicularly to the diffraction plan at distance $L$ that verifies the Fraunhofer criterion of propagation also known as far field region $L>>2 a^{2} / \lambda$, the expression of the field $E_{1}$ is obtained by the following equation:

$$
E_{1}=\xi \int_{\frac{-a}{2}}^{\frac{a}{2}} e^{-j k x \sin (\theta)} d x=\xi a \sin c\left(\frac{\pi a x}{\lambda L}\right)
$$

Where $\xi=E_{0} r^{-1} e^{j(\omega t-\varphi)}$ in $\mathrm{V} / \mathrm{m}^{2} . E_{0}$ is the amplitude of the field in $\mathrm{V} / \mathrm{m}, \omega=2 \pi \mathrm{V}$ is the angular frequency in $\operatorname{rad} . \mathrm{Hz}, \varphi$ is the initial phase, $\lambda$ is the wavelength in $\mathrm{m}, k=2 \pi \lambda^{-1}$ is the wave number and $r$ is the radius of propagation from the aperture to a point $x$ on the screen. The expression is obtained using Gauss approximation also known as small angle approximation:

$$
\sin (\theta) \approx \tan (\theta)=\frac{x}{L}
$$

Similarly, the diffraction field from the second aperture has the same expression with additional phase expression:

$$
E_{2}=\xi e^{-j k d \sin (\theta)} \int_{-d-\frac{a}{2}}^{-d+\frac{a}{2}} e^{-j k x \sin (\theta)} d x=\xi a \sin c\left(\frac{\pi a x}{\lambda L}\right) e^{-j k d \sin (\theta)}=E_{1} e^{\frac{-j 2 \pi d x}{\lambda L}}
$$

The interference between the two diffracted fields can be examined by means of second order correlation function which is a function that is proportional to the intensity, over a period of time $T$. The intensity function is calculated based on the superposition of the elementary fields $E=E_{1}+E_{2}$, it is given by the following expression:

$$
I(x)=\frac{1}{2} \varepsilon_{0} c<E E^{*}>=\varepsilon_{0} c|\xi|^{2} a^{2} \sin c^{2}\left(\frac{\pi a x}{\lambda L}\right)\left(1+\cos \left(\frac{2 \pi d x}{\lambda L}\right)\right)
$$

Where $\varepsilon_{0} \approx 8.85 \times 10^{-12} \mathrm{~F} / \mathrm{m}$ is the permittivity, $c \approx 3 \times 10^{8} \mathrm{~m} / \mathrm{s}$ is the phase speed, $(.)^{*}$ is the conjugate of complex number and $\langle f\rangle$ is the time average operator defined by the equation: 
$<f>=\frac{1}{T} \int_{0}^{T} f(t) d t$

The expression of the intensity can also be written in the following traditional form:

$I(x)=2 I_{0} \sin c^{2}\left(\frac{\pi a x}{\lambda L}\right)\left(1+\cos \left(\frac{2 \pi d x}{\lambda L}\right)\right)$

where $I_{0}=\varepsilon_{0} c E_{0}^{2} a^{2} /\left(2 r^{2}\right)$ in $\mathrm{W} / \mathrm{m}^{2}$. The intensity function is a characterized by two properties, the first is related to the amplitude variation that is modeled by the intensity of diffracted single aperture, the second property is that the interference pattern generated by interaction of the two fields $E_{1}$ and $E_{2}$ by theirs relative phase difference, as $I(x)$ is dependent on the phase difference between the two fields, the introduction of any phase shifting optical device in front of the apertures change the shape of $I(x)$. As a contribution in this paper, we study a linear variation model of the phase difference where we present in the next section the effect of the birefringence on the intensity function.

\section{Interferences using Birefringent Wedge}

The birefringence is the optical property of anisotropic material that is based on polarization, when an incident field passes through a birefringent material, it divides into two parts. Based on this effect, the diffraction model presented in the previous section, changes if we place in front of one the apertures a birefringent material. In this section we study the theoretical intensity function of diffraction by two apertures where a birefringent wedge is placed in front of one of the apertures. We consider a simple case of wedge having slow and ordinary axis. The wedge is characterized by a base of length $a$ which is also the width of the aperture as illustrated in Figure 1.

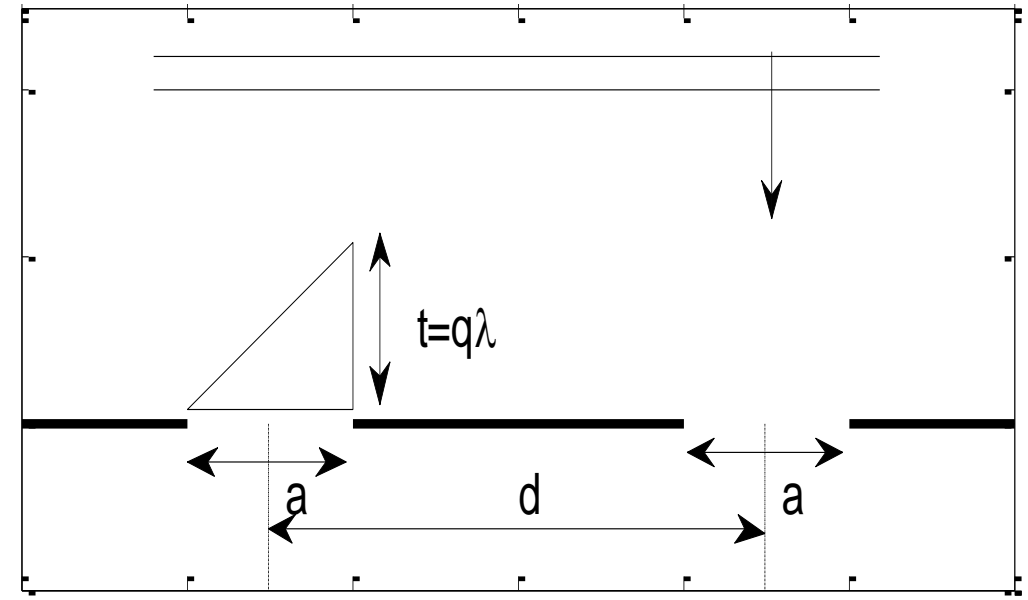

Figure 1. Diffraction by Two Thin Apertures and Birefrengent Wedge with Thickness $t=q \lambda$.

The height or the thickness $t(x)$ is modeled by linear function, for simplicity we consider that at $x=-a / 2$, the thickness is zero and at $x=a / 2$, it equals a multiple of wavelength $q \lambda$ where $q \in R$, this model is described by the following relations: 


$$
\begin{cases}t(x) & =\alpha x+\beta \\ t(-a / 2) & =0 \\ t(a / 2) & =q \lambda\end{cases}
$$

The introduced phase difference by the wedge is given by the relation [10]:

$\phi(x)=\frac{2 \pi}{\lambda} \Delta n t(x)$

With birefringence $\Delta n=\left(n_{e}-n_{o}\right)$. Inserting the linear expression of the thickness yields the following expression of the phase difference:

$\phi(x)=\frac{2 \pi \Delta n q}{a} x+\pi \Delta n q$

If we study the general case of incident field where it can be circularly, elliptically or partially polarized [11], the problem can be treated using Jones formalism [12] and coherency matrix, so the polarization matrix of the wedge is given by the following matrix:

$M=\left(\begin{array}{ll}e^{j \phi(x)} & 0 \\ 0 & 1\end{array}\right)$

Given an incident field $E \in R^{2 \times 1}$ that passes through the material, the output field $E^{\prime}$ is obtained by the linear relation $E^{\prime}=M E$, the new intensity is calculated by the operation:

$I^{\prime}=\frac{\varepsilon_{0} c}{2}<E^{\prime} E^{\prime *}>=\frac{\varepsilon_{0} c}{2} M<E E^{*}>M^{+}=M I M^{+}$

Where (.) ${ }^{+}$denotes the conjugate transpose operator. We begin the analysis of the intensity function by the diffracted field of the left aperture containing the wedge, the expression of the electric field contains the phase difference introduced by the wedge:

$E_{1}=\xi e^{j \pi \Delta n q} \int_{\frac{-a}{2}}^{\frac{a}{2}} e^{-j x\left(k d \sin (\theta)+\frac{2 \pi \Delta n q}{a}\right)} d x=\xi a e^{j \pi \Delta n q} \sin c\left(\frac{\pi a x}{\lambda L}+\pi \Delta n q\right)$

For the second aperture, the field contained the phase difference due to the distance $d$ :

$E_{2}=\xi a \sin c\left(\frac{\pi a x}{\lambda L}\right) e^{-j \frac{2 \pi x d}{\lambda L}}$

The new intensity function of the total field $E=E_{1}+E_{2}$ depends on two variables $x$ and $q$, developing the expression of the intensity function yields the following result: 
$I(x, q)=I_{0}\left(\sin c^{2}(A+\pi \Delta n q)+\sin c^{2}(A)+2 \sin c(A+\pi \Delta n q) \sin c(A) \cos (B+\pi \Delta n q)\right)$

Where the constants $A$ and $B$ are defined by :

$$
\left\{\begin{array}{c}
A=\frac{\pi a x}{\lambda L} \\
B=\frac{2 \pi d x}{\lambda L}
\end{array}\right.
$$

Given the two variables $x$ and $q$ we study some properties of the intensity function. The first property is the intensity at the origin of the Cartesian reference $x=0$, for an arbitrary value of $q$, the intensity at the origin is written as:

$$
I(x=0, q)=I_{0}\left(\sin c^{2}(\pi \Delta n q)+1+2 \sin c(\pi \Delta n q) \cos (\pi \Delta n q)\right)
$$

The second property is related to the verification of the function in the absence of the birefringent wedge, which is equivalent to the condition $q=0$, in this case, in the intensity function is reduced to the standard form presented in equation (4), the result is given by:

$I(x, q=0)=2 I_{0} \sin c^{2}\left(\frac{\pi a x}{\lambda L}\right)\left(1+\cos \left(\frac{2 \pi d x}{\lambda L}\right)\right)$

From the above relation, the intensity at the center in the absence of the wedge is $I(x=0, q=0)=4 I_{0}$, indeed, if we study the problem of diffraction by $N$ apertures, the maximum intensity is $I(x=0)=I_{0} N^{2}$. The fourth property is related to the shape of the intensity distribution for larger values of wedge's thickness, when $q$ becomes larger than the wavelength $\lambda$, we can remark that the intensity $I(x, q)$ near the origin is approximately reduced into the function $I_{0} \sin c^{2}\left(\frac{\pi a x}{\lambda L}\right)$ because $\pi \Delta n q \gg>$.

\section{Simulation Results}

We conduct some computer simulations to verify the effect of birefringent wedge on the intensity distribution of diffraction by two apertures. The parameters of the simulation are described as follows; we consider a $\mathrm{HeNe}$ laser source with wavelength $\lambda=0.633 \mu \mathrm{m}$ and initial intensity of $I_{0}=0.5 \mathrm{~mW} / \mathrm{m}^{2}$. The width of the apertures is set to $a=4 \lambda=2.532 \mu \mathrm{m}$ and the distance between them is $d=40 \lambda=25.32 \mu \mathrm{m}$. The observation screen is placed at distance $L=0.5 \mathrm{~m}$ perpendicularly to the optical axis. The intensity function is sampled in the range $\left[x_{\min }=-10 \mathrm{~cm}, x_{\max }=10 \mathrm{~cm}\right]$.

After testing the program with several values of the thickness $q$ of the birefringent wedge where the chosen value of the birefringence is $\Delta n=10^{-2}$, we present in Figure 2 the obtained results of the intensities. 

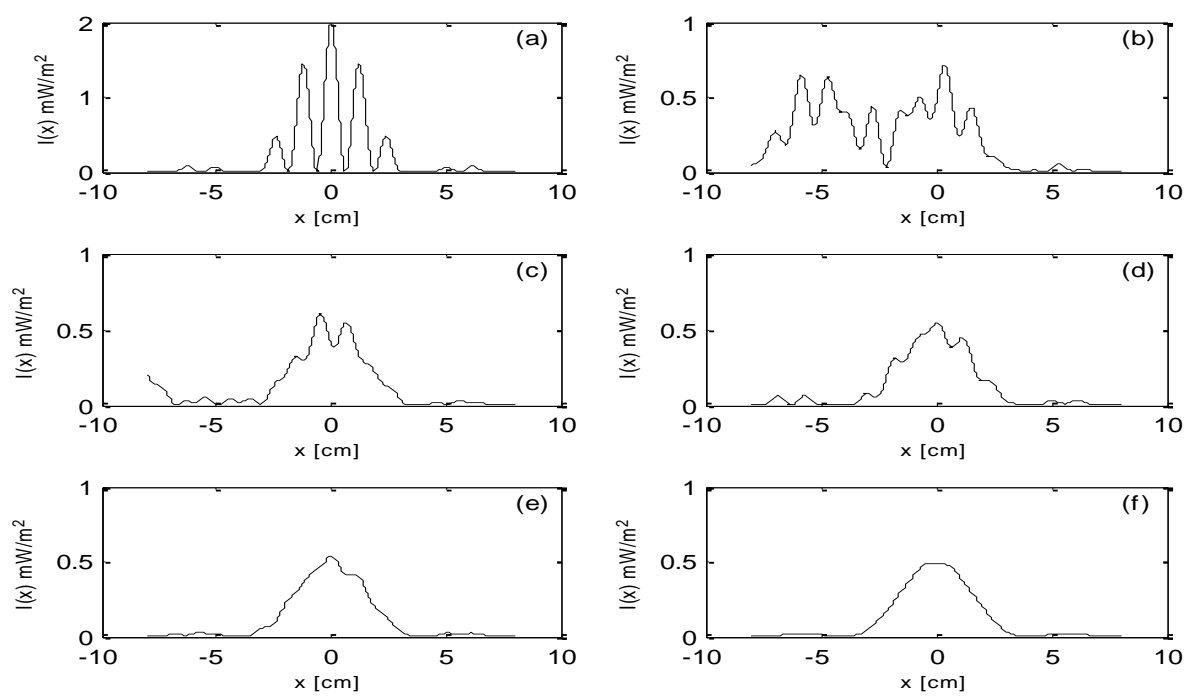

Figure 2. Diffraction by Two Apertures and One Birefringent Wedge with Different Values of Thickness, (a) $I(x, q=0)$, (b) $I(x, q=40)$, (c) $I(x, q=80)$, (d) $I(x, q=100)$, (e) $I(x, q=200)$, and (f) $I(x, q=1000)$

We remark from the figure that the first function (a) represents the intensity in the absence of the wedge $q=0$, the maximum of the intensity is $4 I_{0}=2 \mathrm{~mW} / \mathrm{m}^{2}$ where the amplitude is modeled by the shape of single aperture diffraction. For the cases represented by (b),(c),(d) and (e) we remark that $I(x, q)$ is characterized by fluctuations and non symmetrical effects of the shape, finally for larger values of thickness as represented by the case (e) where $q=1000$, the intensity converges to the shape of single diffraction function with maximum intensity $I_{0}$ where the interference effect diminishes.

We present in Figure.3, the standard diffraction pattern without the birefringent wedge where the amplitude of the diffracted field is modulated by the shape of the single aperture diffraction.

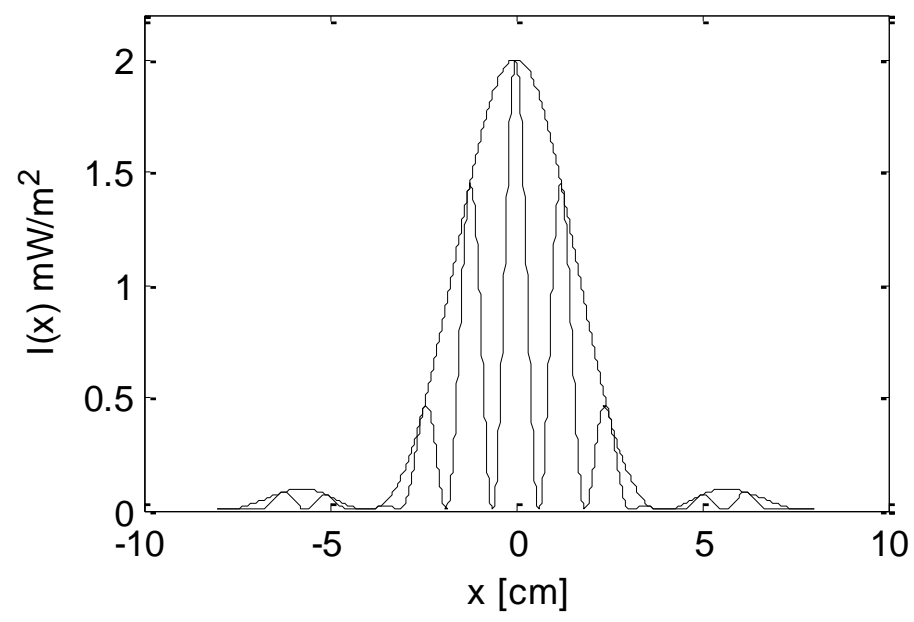

Figure 3. Standard Diffraction Pattern by Two Apertures Illustrating the Amplitude Modulation 
From these results we conclude that the introduction of the birefringent wedge with larger thickness relatively to the wavelength of incident single mode field that is vertically polarized, minimizes the interference effect between the two diffracted fields.

As perspective, we can study a simple extension to the above model by considering a wedge whose length covers the total length of the diffraction plan which is equal to $a+d$, the geometry of this second configuration is described in Figure 4.

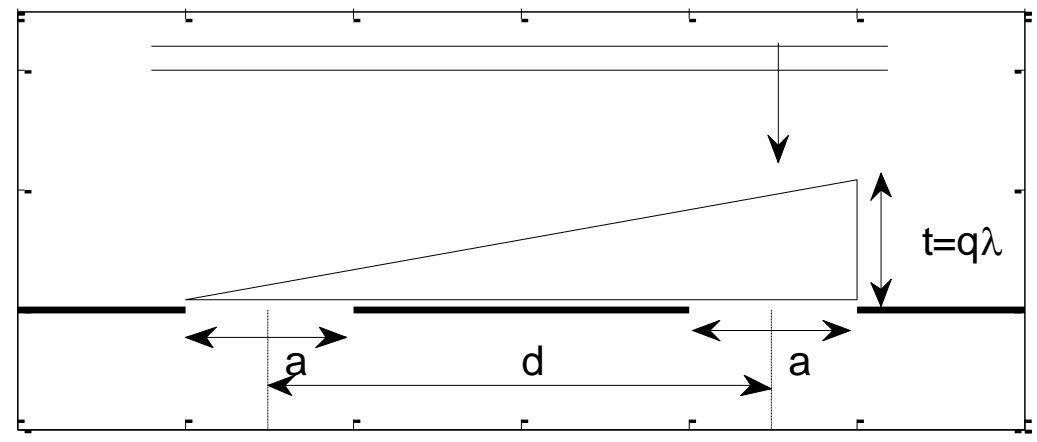

\section{Figure 4. Diffraction by Two Thin Apertures and Birefringent Wedge with Length $a+d$}

Similarly to the previous case, using the boundary conditions, $t(-a / 2)=0$ and $t(a / 2+d)=q \lambda$, the phase difference introduced by the wedge is given by the equation:

$\phi(x)=\frac{2 \pi \Delta n q}{a+d} x+\frac{\pi \Delta n a q}{a+d}$

Following the same method for the computation, the diffracted electric field from the left aperture is:

$E_{1}=\xi \int_{\frac{-a}{2}}^{\frac{a}{2}} e^{-j(k x \sin (\theta)-\phi(x))} d x=\xi a \exp \left(-j \frac{\pi \Delta n a q}{a+d}\right) \sin c\left(\frac{\pi a x}{\lambda L}+\frac{\pi \Delta n a q}{a+d}\right)$

Next, Solving the integral for the right aperture from $d-a / 2$ to $d+a / 2$ gives the following result:

$E_{2}=\xi a \exp \left(-j \frac{\pi \Delta n a q}{a+d}\right) \sin c\left(\frac{\pi a x}{\lambda L}+\frac{\pi \Delta n a q}{a+d}\right) \exp \left(-j\left(\frac{2 \pi d x}{\lambda L}+\frac{2 \pi \Delta n d q}{a+d}\right)\right)$

The resulting intensity is therefore given by:

$I(x, q)=2 I_{0} \sin c^{2}\left(\frac{\pi a x}{\lambda L}+\frac{\pi \Delta n a q}{a+d}\right)\left[1+\cos \left(\frac{2 \pi d x}{\lambda L}+\frac{2 \pi \Delta n d q}{a+d}\right)\right]$

We remark that if $q=0$ which refers to the case of absence of the wedge, we obtain the standard expression of double-aperture. 


\section{Conclusion}

In this paper, we have studied the problem of far field diffraction of single mode laser by two thin apertures using small angle approximation with the introduction of birefringent material. The parameters of the birefringent wedge have an impact on the intensity distribution. We have studied some properties of the intensity where a birfringent wedge is placed in front of one of the apertures. We have demonstrated theoretically and numerically that larger values of wedge's thickness relatively the wavelength changes the diffraction pattern such that the interference pattern is minimized.

\section{Appendix}

1. As mentionned in the introduction, the width of the aperture has an impact on the shape of the diffraction, similarly to the frequency analysis of a waveform, the spectrum is a function of the width of the observation window, as an illustrative example we present a simple case of periodic waveform $x(t)=e^{j 2 \pi f_{0} t}$.We consider the case $t \in R$, the spectrum is given by:

$$
X(f)=\int_{R} x(t) e^{-j 2 \pi f t} d t=\delta\left(f-f_{0}\right)
$$

Next a consider that the variable $t \in[-T / 2, T / 2]$, equivalently, the waveform can be written as $x(t)=e^{j 2 \pi f_{0} t} r(t)$ where the introduced function is defined by $r(t)=1$ if $|t|<T / 2$ and equals zero otherwise. The Fourier transform of this function is $r(f)=T \sin c(\pi f T)$. Using the convolution theorem, the spectrum of $x(t)$ is calculated by the following equation:

$$
X(f)=\delta\left(f-f_{0}\right) * T \sin c(\pi f T)=T \sin c\left(\pi T\left(f-f_{0}\right)\right)
$$

Where $*$ denotes the convolution product. We remark that the spectrum depends on the frequency response of the introduced function $r(t)$.

2. We present, in this part, the Mathworks program to generate the results of the diffraction, the output vectors are I for standard double aperture intensity and I 2 for the configuration of the birefringent wedge.

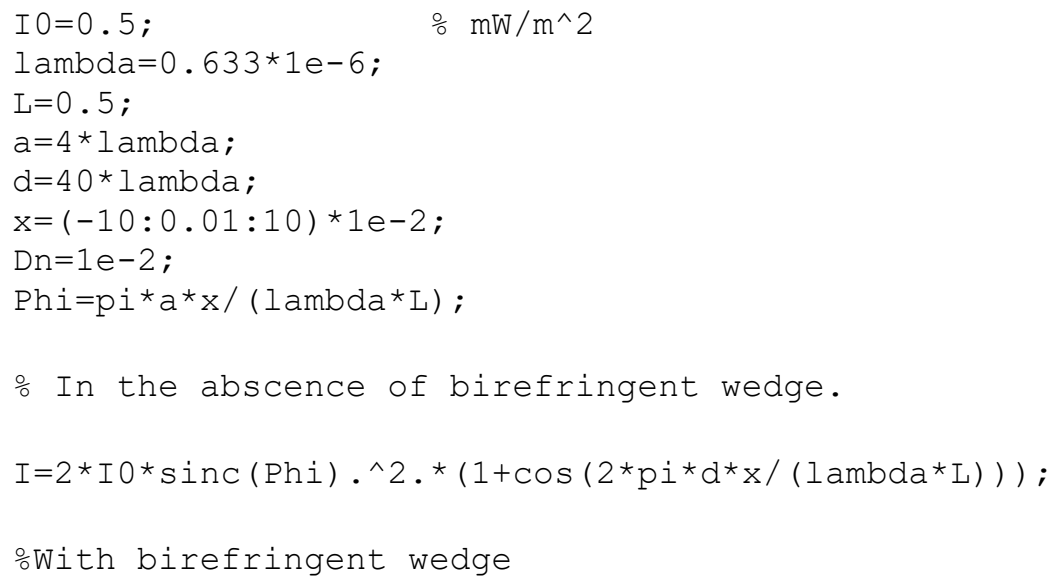


$\operatorname{Phi}=2 * \mathrm{pi}{ }^{\star} \mathrm{d}^{\star} \mathrm{x} /(\mathrm{l}$ ambda*L)

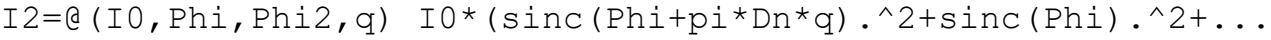
$\left.2{ }^{\star} \operatorname{sinc}\left(\mathrm{Ph} i+\mathrm{pi}{ }^{\star} \mathrm{Dn}{ }^{\star} \mathrm{q}\right) .{ }^{*} \operatorname{sinc}(\mathrm{Ph} i) .{ }^{\star} \cos \left(\mathrm{Ph} i 2+\mathrm{pi}{ }^{*} \mathrm{Dn}{ }^{\star} \mathrm{q}\right)\right)$;

plot (x, I2 (I0,Phi, Phi2,100));

\section{References}

[1] L. Rayleigh, "Note on the Remarkable Case of Diffraction Spectra Described by Prof. Wood", Philos. Mag., vol. 14, no. 60, (1907)

[2] J. W. Goodman, "Statistical Optics", Wiley Classics Library, ISBN 0-47 1-0 1502-4, (2000).

[3] S. Kocsis, "Observing the average trajectories of single photons in a two-slit interferometer," 2011 International Quantum Electronics Conference (IQEC) and Conference on Lasers and Electro-Optics (CLEO) Pacific Rim incorporating the Australasian Conference on Optics, Lasers and Spectroscopy and the Australian Conference on Optical Fibre Technology, Sydney, NSW, (2011), pp. 105-107.

[4] R. Bach, New J. Phys. 15 033018, (2013).

[5] J. Davis, J. Adachi, C. Fernández-Pousa and I. Moreno, "Polarization beam splitters using polarization diffraction gratings", Opt. Lett., vol. 26, (2001), pp. 587-589.

[6] E. Hecht, Optics (4th ed.), (2001), pp. 352-5.

[7] K. Oka and T. Kaneko, "Compact complete imaging polarimeter using birefringent wedge prisms", Opt. Express, vol. 11, (2003), pp. 1510-1519.

[8] M. Teresa, "Image formation through birefringent uniaxial wedges", Pure and Applied Optics: Journal of the European Optical Society Part A, (1996).

[9] V. P. Vasiliev, "Retroreflectors using a birefringent wedge for efficient velocity aberration compensation", 2016 International Conference Laser Optics (LO), St. Petersburg, (2016), pp. R6-9-R6-9.

[10] J. Ma, L. Pei, C. Xu and Y. Yao, "Real-time polarization state measurement based on a birefringent crystal wedge", Journal of Modern Optics, vol. 63, no. 21, (2016).

[11] R. Bhandari, "Polarization of light and topological phases", Physics Reports, ISSN 0370-1573, vol. 281, no. 1, (1997), pp. 1-64.

[12] H. Günhan Akarçay, A. Hohmann, A. Kienle, M. Frenz and J. Ricka, "Monte Carlo modeling of polarized light propagation: Stokes vs. Jones. Part I”, Appl. Opt., vol. 53, (2014), pp. 7576-7585. 
International Journal of Advanced Science and Technology

Vol.114 (2018) 